\title{
Peroxisome Proliferator-activated Receptor-gamma Inhibits the Activation of STAT3 in Cerulein-stimulated Pancreatic Acinar Cells
}

\author{
Kyung Don Ju', Joo Weon Lim², Hyeyoung Kim ${ }^{1,2}$ \\ ${ }^{1}$ Department of Pharmacology, Yonsei University College of Medicine, ${ }^{2}$ Department of Food and Nutrition, Brain Korea 21 PLUS Project, College \\ of Human Ecology, Yonsei University, Seoul, Korea
}

\begin{abstract}
Cerulein-induced pancreatitis is similar to human edematous pancreatitis, characterized by the dysregulation of digestive enzyme production, edema formation, and an infiltration of inflammatory cells into the pancreas. We previously showed that the Janus kinase 2 (JAK2)/STAT3 pathway mediates inflammatory signaling in cerulein-stimulated pancreatic acinar cells. PPAR- $\gamma$ has been implicated in the regulation of inflammatory responses in several cells. In the present study, we investigated the role of PPAR- $\gamma$ in cerulein-induced activation of JAK2/STAT3 in pancreatic acinar cells. Treatment with cerulein induced the activation of JAK2/STAT3 and PPAR- $\gamma$ expression in AR42J cells. Cerulein-induced PPAR- $\gamma$ expression was inhibited by AG490, a JAK2/STAT3 inhibitor, in AR42J cells. An immunoprecipitation analysis showed that PPAR- $\gamma$ binds to STAT3 in cerulein-stimulated AR42J cells. Down-regulation of PPAR- $\gamma$ by siRNA increased STAT3 phosphorylation in AR42J cells stimulated with cerulein. These results show that PPAR- $\gamma$ inactivates STAT3 by directly interacting with STAT3 in cerulein-stimulated pancreatic acinar cells. Overexpression of PPAR- $\gamma$ may be beneficial for preventing pancreatitis by suppressing the activation of STAT3 in pancreatic acinar cells.
\end{abstract}

(J Cancer Prev 2017;22:189-194)

Key Words: Acinar cells, Cerulein, Pancreatitis, PPAR- $\gamma$, Signal transducers and activators of transcription3

\section{INTRODUCTION}

Acute pancreatitis is a multifactorial inflammatory disease involving the release of digestive enzymes to the pancreatic interstitium and the systemic circulation as well as increased cytokine production, leading to deleterious local and systemic effects. ${ }^{1}$ High doses of cerulein, a cholecystokinin analogue, result in experimental pancreatitis, which is characterized by an elevation in serum levels of digestive enzymes, cytoplasmic vacuolization, and edema formation, similar to the characteristics of human pancreatitis. ${ }^{2}$

The Janus kinase (JAK)/STAT3 pathway is involved in the actions of primarily non-immune mediators, such as growth factors and hormones, as well as the immune response of numerous cytokines. STAT target genes include cytokines, adhesion molecules, and other inflammatory mediators. ${ }^{3}$ We previously demonstrated that cerulein induces the JAK2/STAT3 signaling pathway in pancreatic acinar cells and in the rat pancreas. ${ }^{4}$

PPAR- $\gamma$ is a member of the nuclear hormone receptor superfamily and plays a pivotal role in promoting fibroblast differentiation into adipocytes. ${ }^{5}$ PPAR- $\gamma$ ligands, such as 15 -deoxy-delta-12,14-prostaglandin $\mathrm{J} 2$ and troglitazone, inhibit inflammatory responses by inhibiting chemokine expression in human tubular epithelial cells. ${ }^{6}$ Synthesized PPAR- $\gamma$ ligands, including thiazolidinedione derivatives and troglitazone, reduce inflammation by modulating the production of inflammatory mediators in experimental colitis. ${ }^{7}$ Pioglitazone, a PPAR- $\gamma$ activator, attenuates the severity of acute pancreatitis. ${ }^{8}$ However, recent studies have reported that thiazolidinedione derivatives

Received June 13, 2017, Revised August 10, 2017, Accepted August 12, 2017

Correspondence to: Hyeyoung Kim

Department of Food and Nutrition, College of Human Ecology, Yonsei University, 50 Yonsei-ro, Sedaemun-gu, Seoul 03722, Korea

Tel: +82-2-2123-3125, Fax: +82-2-364-5781, E-mail: kim626@yonsei.ac.kr, ORCID: Hyeyoung Kim, http://orcid.org/0000-0002-7019-917X

Copyright (C) 2017 Korean Society of Cancer Prevention

(c) This is an Open Access article distributed under the terms of the Creative Commons Attribution Non-Commercial License (http://creativecommons.org/icenses/by-nc/4.0) which permits unrestricted non-commercial use, distribution, and reproduction in any medium, provided the original work is properly cited. 
have PPAR- - -independent effects depending on the cell type and response. ${ }^{9.10}$ Therefore, it is necessary to clarify the direct effect of PPAR- $\gamma$ on inflammatory signaling to determine its role in the pathophysiology of pancreatitis. In the present study, we down-regulated PPAR- $\gamma$ using siRNA to investigate its role in JAK2/STAT3 inflammatory signaling in cerulein-stimulated pancreatic acinar AR42J cells. To determine the role of JAK2/STAT3 in the expression of PPAR- $\gamma$, cerulein-stimulated AR42J cells were pretreated with AG490, a JAK2/STAT3 inhibitor. ${ }^{11}$

\section{MATERIALS AND METHODS}

\section{Cell line and culture condition}

Rat pancreatic acinar cells (AR42J cells) were obtained from the American Type Culture Collection (Manassas, VA, USA). Cells were cultured in Dulbecco's modified Eagle's medium (Sigma, St. Louis, MO, USA) supplemented with 10\% FBS (GIBCO-BRL, Grand Island, NY, USA) and $1 \%$ antibiotics (100 units/mL penicillin and $100 \mu \mathrm{g} / \mathrm{mL}$ streptomycin). Cells were used for experiments after incubation for 20 to 24 hours at $37^{\circ} \mathrm{C}$ in a humidified atmosphere of $90 \%$ air and $10 \% \mathrm{CO}_{2}$.

\section{Experimental protocol}

Cells were stimulated with cerulein $\left(10^{-8} \mathrm{M}\right)$ at various time points (0, 30, 60, 120, and 240 minutes) to evaluate JAK2/STAT3 phosphorylation, PPAR- $\gamma$ expression, and interactions between PPAR $\gamma$ and STAT3 in cerulein-stimulated AR42J cells. For immunofluorescence staining, the cells were stimulated with cerulein $\left(10^{-8} \mathrm{M}\right)$ for 2 hours on Lab-TeK chamber slide glasses (Labtek, Scotts Valley, CA, USA). To examine the effect of AG490 on the expression of PPAR- $\gamma$, cells were pretreated with the inhibitor of JAK2/STAT3, AG490 (40 $\mu$ M; Santa Cruz Biotechnology, Dallas, TX, USA) or the inhibitor of protein biosynthesis, cycloheximide (100 $\mu \mathrm{g} / \mathrm{mL}$, Catalog No. C7698; Sigma), for 1 hour and then stimulated with cerulein $\left(10^{-8} \mathrm{M}\right)$ for 2 hours. PPAR- $\gamma$ expression was determined by Western blotting. To evaluate the direct effect of PPAR- $\gamma$ on JAK2/STAT3 activation, the cells were transfected with siRNA directed against PPAR- $\gamma$ or the negative control (N/C) using Lipofectamine 2000 (Invitrogen, Waltham, MA, USA) for 48 hours and then stimulated with cerulein $\left(10^{-8} \mathrm{M}\right)$ for 2 hours. JAK2/STAT3 phosphorylation and PPAR- expression were determined in the transfected cells.

\section{Western blot analysis}

Whole cell extracts were prepared using a previously described method. ${ }^{4}$ The whole cell extracts were separated by $8 \%$ SDS PAGE under reducing conditions and transferred to nitrocellulose membranes (Amersham Inc., Arlington Heights, IL, USA) by Western blot analysis. The transfer of the protein and equal loading in the lanes were verified by reversible staining with Ponceau S. The membranes were blocked with $5 \%$ non-fat dry milk in TBS and $0.15 \%$ Tween 20 (TBS-T) for 3 hours at $15^{\circ} \mathrm{C}$ to $25^{\circ} \mathrm{C}$. The proteins were detected with polyclonal antibodies for JAK2 (sc-7229; Santa Cruz Biotechnology, Santa Cruz, CA, USA), STAT3 (Cat. No. 06-596; Upstate Biotechnology, Lake Placid, NY, USA), phospho-JAK2 (Cat. No. 3771; Cell Signaling Technology, Beverly, MA, USA), phospho-STAT3 (Cat. No. 9131; Cell Signaling Technology), and PPAR- $\gamma$ (sc-7273, Santa Cruz Biotechnology) diluted in TBS-T containing $5 \%$ dry milk, and incubated at $4^{\circ} \mathrm{C}$ overnight. After washing in TBS-T, the immunoreactive proteins were visualized using goat anti-rabbit secondary antibodies (sc-2004; Santa Cruz Biotechnology) conjugated to horseradish peroxidase by enhanced chemiluminescence (Amersham Inc.). To quantify changes in protein levels, the protein bands were scanned using a BioRad scanner (GS-700; Bio-Rad Laboratories, Hercules, CA, USA) driven by volume analysis software, and quantified with Molecular Analysis software (version 1.4). The protein level was compared to that of the loading control actin, total JAK2, or total STAT3 and expressed as a percentage density ratio. The protein concentration was determined using the Bradford method.

\section{Small interfering RNA (siRNA) targeting PPAR- $\gamma$}

To down-regulate PPAR- $\gamma$, siRNA was designed to target the PPAR- $\gamma$ gene using BLOCK-iT ${ }^{\mathrm{TM}}$ RNAi Designer and synthesized by Invitrogen (Carlsbad, CA, USA). Stealth ${ }^{\mathrm{TM}}$ RNAi Negative Control Duplexes were used as a non-targeted control. siRNA was transfected into cells using Lipofectamine 2000 by incubation for 48 hours.

\section{Co-immunoprecipitation}

The whole cell extracts were incubated with a specific antibody and protein $\mathrm{G} / \mathrm{A}$, followed by immobilization on agarose overnight at $4^{\circ} \mathrm{C}$ in radioimmunoprecipitation assay (RIPA) buffer containing $25 \mathrm{mM}$ Tris-HCl, pH 7.5, $150 \mathrm{mM} \mathrm{NaCl}, 1 \%$ Triton X-100, 1 mM EDTA, 1 mM EGTA, 1 mM phenyl-methyl-sulfonyl fluoride, $0.25 \% \mathrm{NP}-40$, and $0.5 \%$ sodium deoxycholate. The protein $\mathrm{G} / \mathrm{A}$-antibody-antigen complex was collected by washing three times with ice-cold RIPA buffer. The final pellet was resuspended with $50 \mu \mathrm{L}$ of SDS-sample buffer and boiled for 5 minutes. This preparation was subjected to Western blotting with specific antibodies. 


\section{Immunofluorescence staining}

The cells were stimulated with cerulein for 2 hours on Lab-TeK chamber slide glasses, and fixed with cold acetone. After air drying and washing with PBS three times, the cells were incubated with primary antibodies (final concentration, $2 \mu \mathrm{g} / \mathrm{mL}$ ) for 1 hour at room temperature. For double immunofluorescence staining, monoclonal mouse anti-PPAR- $\gamma$ and polyclonal rabbit anti-STAT3 were used simultaneously as primary antibodies. The cells were washed for 5 minutes six times with PBS and reacted with secondary antibodies for 1 hour at room temperature.
Rhodamine-labeled mouse anti-rabbit immunoglobulin G (IgG) antibody (final concentration, $2 \mu \mathrm{g} / \mathrm{mL}$ ) and fluorescein isothiocyanate (FITC)-labeled donkey anti-mouse IgG antibody (final concentration, $2 \mu \mathrm{g} / \mathrm{mL}$ ) were used simultaneously. After removal of the secondary antibodies, the cells were washed six times with PBS for 5 minutes each wash and covered with the anti-fade medium Vectashield containing 4',6-diamidino-2-phenylindole. The cells stained with rhodamine- and/or FITC-labeled antibody were examined with a laser scanning confocal microscope (Leica TCS-NT, Heidelberg, Germany) and photographed. Optical filters used for excitation were 450 to $490 \mathrm{~nm}$ for FITC and 510 to 560
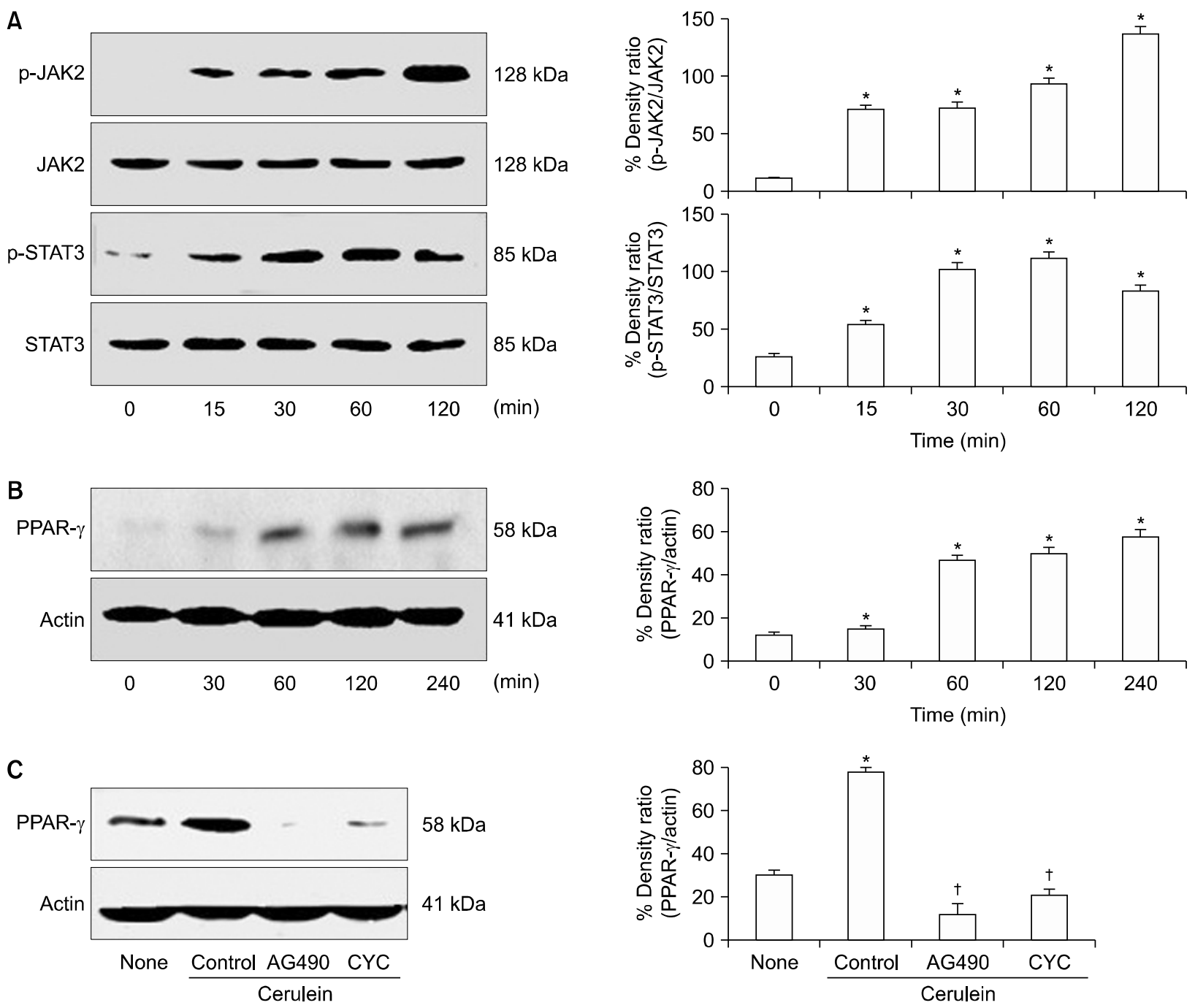

Figure 1. The levels of JAK2/STAT3 and PPAR- $\gamma$ in cerulein-stimulated AR42J cells with or without AG490 or cycloheximide (CYC). (A, B) The cells were stimulated with cerulein $\left(10^{-8} \mathrm{M}\right)$ at various time points. (C) The cells were pretreated with AG490 (40 $\left.\mu \mathrm{M}\right)$ or CYC (100 $\mu \mathrm{g} / \mathrm{mL})$ for 1 hour and then stimulated with cerulein $\left(10^{-8} \mathrm{M}\right)$ for 2 hours. The specific protein levels in the whole cell extracts were determined by Western blot analysis and expressed as a percentage density ratio. The phosphorylated levels of JAK2 and STAT3 proteins were compared to the respective total protein levels (A), and PPAR- $\gamma$ protein levels were normalized with those of the loading control actin (B, C). All values are expressed as means \pm SEM of four independent experiments. ${ }^{*} P<0.05$ vs. none (untreated cells); ${ }^{\dagger} P<0.05$ vs. control (cells treated with cerulein alone). 
nm for rhodamine. The regions stained with rhodamine- or FITC-labeled secondary antibody were detected as red or green, respectively. The overlap between rhodamine (in red) and FITC (in green) showed STAT3/PPAR- $\gamma$.

\section{Statistical differences}

Statistical differences were determined using one-way ANOVA and Newman-Keul's tests. All values are expressed as means \pm SEM of four independent experiments.

\section{RESULTS}

We investigated the relationship between JAK2/STAT3 activation and PPAR- $\gamma$ expression in AR42J cells. As shown in Figure $1 \mathrm{~A}$, cerulein induced the phosphorylation of JAK2 and
STAT3, but did not affect the levels of total JAK2 and STAT3 in AR42J cells. Cerulein increased the expression of PPAR- $\gamma$ in a time-dependent manner for 2 hours (Fig. 1B). Cerulein-induced PPAR- $\gamma$ expression was inhibited by both the JAK2/STAT3 inhibitor AG490 and the protein biosynthesis inhibitor cycloheximide in AR42J cells during a 2-hour culture period (Fig. 1C). Low levels of PPAR- $\gamma$ can be caused by decreased mRNA expression or increased protein degradation. Since the protein level of PPAR- $\gamma$ decreased in response to cycloheximide, low levels of PPAR $\gamma$ in cells treated with AG490 suggest that JAK2/STAT3 mediates PPAR- $\gamma$ expression at the transcriptional level, not the post-translational level, in cerulein-stimulated pancreatic acinar cells. These results suggest that cerulein-induced expression is mediated by JAK2/STAT3 signaling in pancreatic acinar AR42J cells.
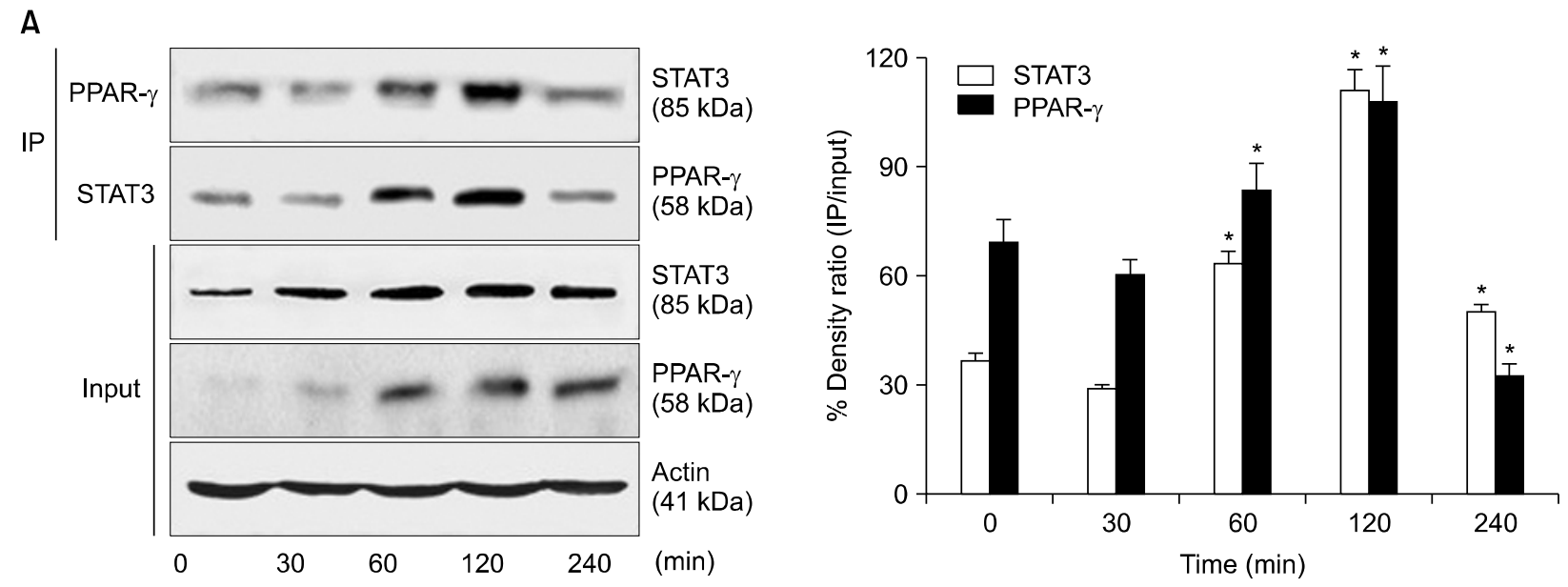

B

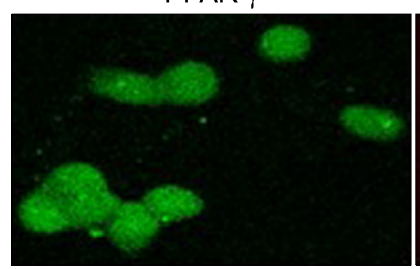

STAT3

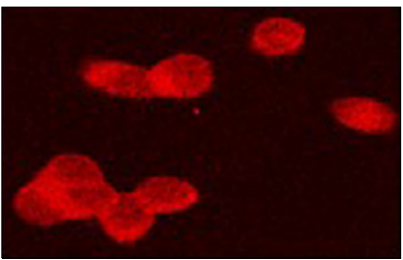

DAPI

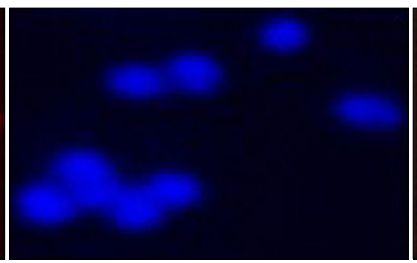

Merge

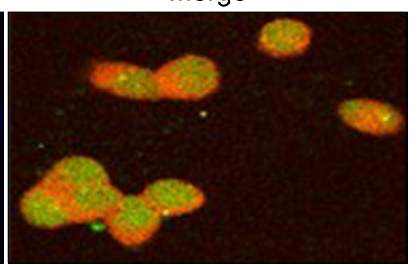

Figure 2. Binding of PPAR- $\gamma$ to STAT3 in cerulein-stimulated AR42J cells. (A) The cells were stimulated with cerulein (10 $\left.{ }^{-8} \mathrm{M}\right)$ for the indicated periods. Whole cell extracts were subjected to immunoprecipitation (IP) with an anti-PPAR- $\gamma$ antibody (first panel) or anti-STAT3 antibody (second panel), followed by Western blotting with an anti-STAT3 antibody or anti-PPAR- $\gamma$ antibody. The samples (input) were subjected to SDS-PAGE and analyzed by Western blot analysis using an anti-STAT3 antibody (third panel), anti-PPAR- $\gamma$ antibody (fourth panel), or anti-actin antibody (fifth panel). The protein level was expressed as the percentage density ratio of immunoprecipitated STAT3 to input STAT3 or immunoprecipitated PPAR $-\gamma$ to input PPAR $-\gamma$. All values are expressed as means \pm SEM of four independent experiments. $* P<0.05$ vs. corresponding 0 hour. (B) Cells were stimulated with cerulein $\left(10^{-8} \mathrm{M}\right)$ for 2 hours on Lab-TeK chamber slide glasses. The cells were fixed with cold acetone, washed with PBS, and then incubated with anti-PPAR- $\gamma$ and anti-STAT3 antibodies for 1 hour. After washing with PBS, the cells were incubated with the secondary antibodies for 1 hour and covered with the Vectashield antifade medium containing 4',6-diamidino-2-phenylindole (DAPI). The region stained with fluorescein isothiocyanate- or rhodamine-labeled secondary antibody was detected as green or red, respectively. PPAR- $\gamma$ (green) and STAT3 (red) were merged on a single screen (Merge). DAPI staining was used for nuclear quantification. 
We investigated whether PPAR- $\gamma$ regulates the activation of JAK2/STAT3 in cerulein-stimulated AR42J cells by examining the binding of PPAR- $\gamma$ to STAT3. As shown in Figure 2A, STAT3 was detected in anti-PPAR- $\gamma$ immunoprecipitates of whole cell extracts from cells stimulated with cerulein. The whole cell extract of the stimulated cells was immunoprecipitated with an anti-STAT3 antibody and then subjected to Western blot analysis using an anti-PPAR $\gamma$ antibody. PPAR- $\gamma$ was detected in the anti-STAT3 immunoprecipitates. As shown in Figure 2B, STAT3 co-localized with PPAR- $\gamma$ in cerulein-stimulated AR42J cells, confirming the interaction of PPAR- $\gamma$ with STAT3. These results demonstrate that PPAR- $\gamma$ directly binds to STAT3 in cerulein-stimulated AR42J cells. As shown in Figure 3, cerulein-induced expression of PPAR- $\gamma$ was down-regulated in cells transfected with PPAR- $\gamma$ siRNA compared to cells transfected with N/C siRNA. However, transfection with PPAR $\gamma$ siRNA enhanced the phosphorylation of STAT3 in cerulein-stimulated cells. PPAR- $\gamma$ siRNA did not affect the phosphorylation of JAK2, total JAK2, or total STAT3 in cerulein-stimulated AR42J cells. These results demonstrate that PPAR- $\gamma$ inactivates STAT3 by direct binding to STAT3 in cerulein-stimulated pancreatic acinar cells.

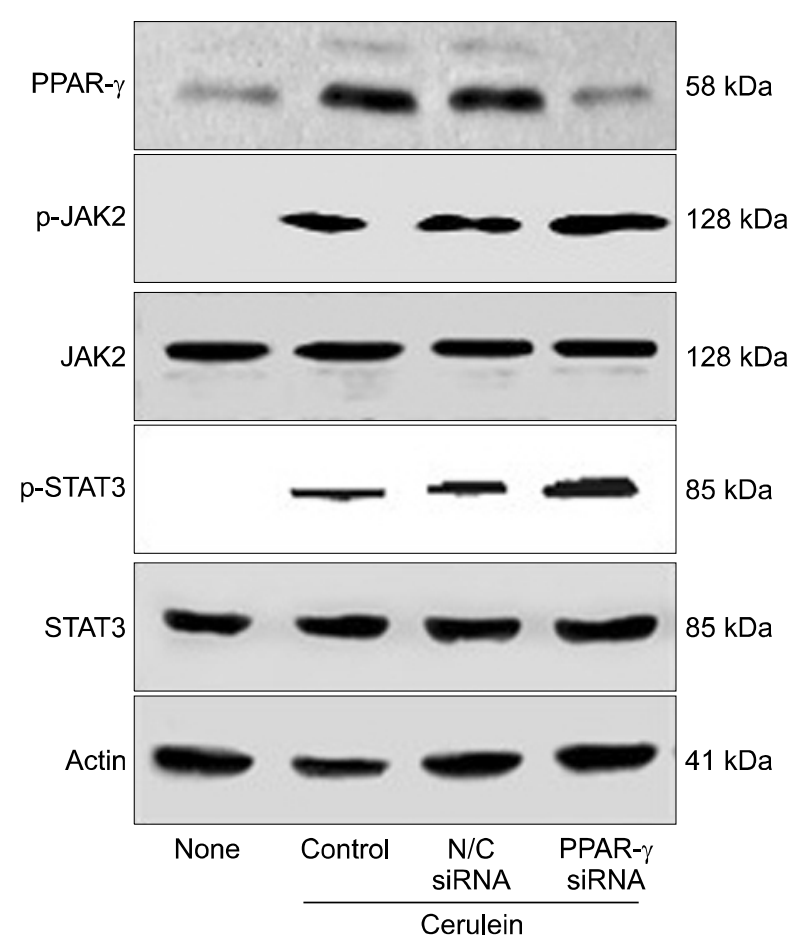

\section{DISCUSSION}

The JAK/STAT pathway mediates a wide variety of biological processes, such as the immune response, differentiation, cell survival, and proliferation. ${ }^{12}$ STAT3 plays an important role in inflammation and tumorigenesis by regulating cell proliferation, differentiation, and metabolism. ${ }^{3,13}$ STAT3 signaling is regulated by transcriptional control, posttranslational modification, and cellular localization. ${ }^{14-16}$ Here, we found that PPAR- $\gamma$ inhibited STAT3 activation by directly binding to STAT3 in cerulein-stimulated cells. Wang et al. ${ }^{17}$ demonstrated that the expression of PPAR- $\gamma$ and its agonists 15-deoxy-delta-12,14-prostaglandin J2 and troglitazone completely abolish IL-6-inducible cell proliferation and induce apoptosis via the expression of multiple cell cycle or apoptosis genes in multiple myeloma cells. They suggest a potential interaction of PPAR- $\gamma$ with STAT3 in multiple myeloma cells. Recently, Ji et al. ${ }^{18}$ showed that the phosphorylation and nuclear translocation of STAT3 are related to the nuclear translocation, DNA binding, and transcriptional activities of PPAR- $\gamma$ in gastric epithelial AGS cells infected with Helicobacter pylori. In the present study, we found that PPAR- $\gamma$ siRNA

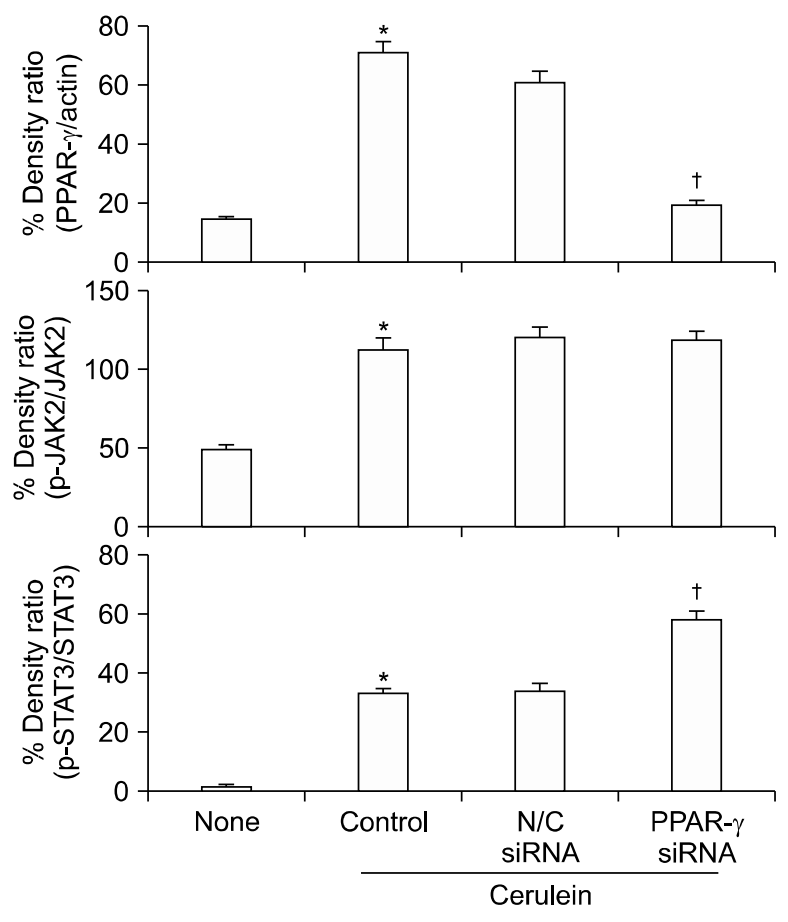

Figure 3. The levels of JAK2/STAT3 and PPAR- $\gamma$ in cerulein-stimulated AR42J cells transfected with PPAR- $\gamma$ siRNA or negative control (N/C). The cells were transfected with siRNA directed against PPAR- $\gamma$ or the N/C using Lipofectamine 2000 (Invitrogen) for 48 hours, and then stimulated with cerulein $\left(10^{-8} \mathrm{M}\right)$ for 2 hours. The levels of total and phospho-specific forms of JAK2 and STAT3, and PPAR- $\gamma$ in the whole cell extracts were determined by Western blot analysis. The protein level was compared to that of total JAK2, total STAT3, or the loading control actin and expressed as the percentage density ratio. All values are expressed as means \pm SEM of four independent experiments. $* P<0.05$ vs. none (untreated cells); ${ }^{\dagger} P<0.05$ vs. N/C siRNA (cells transfected with N/C siRNA and stimulated with cerulein). 
enhanced STAT3 activation, but did not affect JAK2 activation in cerulein-stimulated cells. These results suggest a direct role of PPAR- $\gamma$ in STAT3 activation in cerulein-stimulated pancreatic acinar cells. A novel finding in the present study is that PPAR- $\gamma$, which is up-regulated by cerulein, interacts with and subsequently inactivates STAT3 in pancreatic acinar cells. Overexpression of PPAR- $\gamma$ may be beneficial for preventing inflammation of pancreas by inhibiting the activation of STAT3 in pancreatic acinar cells.

\section{ACKNOWLEDGMENTS}

This work was supported by a grant from the National Research Foundation (NRF) of Korea, which is funded by the Korean Government (NRF-2015 R1A2A2A01004855).

\section{CONFLICTS OF INTEREST}

No potential conflicts of interest were disclosed.

\section{REFERENCES}

1. Granger J, Remick D. Acute pancreatitis: models, markers, and mediators. Shock 2005;24 Suppl 1:45-51.

2. Hofbauer B, Saluja AK, Lerch MM, Bhagat L, Bhatia M, Lee HS, et al. Intra-acinar cell activation of trypsinogen during caerulein-induced pancreatitis in rats. Am J Physiol 1998;275:G352-62.

3. Qi QR, Yang ZM. Regulation and function of signal transducer and activator of transcription 3. World J Biol Chem 2014:5:231-9.

4. Yu JH, Kim KH, Kim H. SOCS 3 and PPAR-gamma ligands inhibit the expression of IL-6 and TGF-betal by regulating JAK2/STAT3 signaling in pancreas. Int J Biochem Cell Biol 2008;40:677-88.

5. Kliewer SA, Lenhard JM, Willson TM, Patel I, Morris DC, Lehmann JM. A prostaglandin J2 metabolite binds peroxisome proliferator-activated receptor gamma and promotes adipocyte differentiation. Cell 1995;83:813-9.

6. Wang WM, Zhang HD, Jin YM, Zhu BB, Chen N. PPAR-gamma agonists inhibit TGF-betal-induced chemokine expression in human tubular epithelial cells. Acta Pharmacol Sin 2009;30:107-12.
7. Celinski K, Dworzanski T, Fornal R, Korolczuk A, Madro A, Brzozowski $\mathrm{T}$, et al. Comparison of anti-inflammatory properties of peroxisome proliferator-activated receptor gamma agonists rosiglitazone and troglitazone in prophylactic treatment of experimental colitis. J Physiol Pharmacol 2013;64:587-95.

8. Wan H, Yuan Y, Liu J, Chen G. Pioglitazone, a PPAR- $\gamma$ activator, attenuates the severity of cerulein-induced acute pancreatitis by modulating early growth response- 1 transcription factor. Transl Res 2012;160:153-61.

9. Liu HB, Hu YS, Medcalf RL, Simpson RW, Dear AE. Thiazolidinediones inhibit TNFalpha induction of PAI-1 independent of PPARgamma activation. Biochem Biophys Res Commun 2005; 334:30-7.

10. Artwohl M, Fürnsinn C, Waldhäusl W, Hölzenbein T, Rainer G, Freudenthaler A, et al. Thiazolidinediones inhibit proliferation of microvascular and macrovascular cells by a PPARgamma-independent mechanism. Diabetologia 2005;48:586-94.

11. Park JS, Lee J, Lim MA, Kim EK, Kim SM, Ryu JG, et al. JAK2-STAT3 blockade by AG490 suppresses autoimmune arthritis in mice via reciprocal regulation of regulatory $\mathrm{T}$ Cells and Th17 cells. J Immunol 2014;192:4417-24.

12. Villarino AV, Kanno Y, Ferdinand JR, O'Shea JJ. Mechanisms of Jak/STAT signaling in immunity and disease. J Immunol 2015;194:21-7.

13. Fang J, Chu L, Li C, Chen Y, Hu F, Zhang X, et al. JAK2 inhibitor blocks the inflammation and growth of esophageal squamous cell carcinoma in vitro through the JAK/STAT3 pathway. Oncol Rep 2015:33:494-502.

14. Hillmer EJ, Zhang H, Li HS, Watowich SS. STAT3 signaling in immunity. Cytokine Growth Factor Rev 2016;31:1-15.

15. Jia Y, Jing J, Bai Y, Li Z, Liu L, Luo J, et al. Amelioration of experimental autoimmune encephalomyelitis by plumbagin through down-regulation of JAK-STAT and NF-KB signaling pathways. PLOS One 2011;6:e27006.

16. Ju KD, Lim JW, Kim KH, Kim H. Potential role of NADPH oxidase-mediated activation of Jak2/Stat3 and mitogen-activated protein kinases and expression of TGF- $\beta 1$ in the pathophysiology of acute pancreatitis. Inflamm Res 2011;60:791-800.

17. Wang LH, Yang XY, Zhang X, Huang J, Hou J, Li J, et al. Transcriptional inactivation of STAT3 by PPARgamma suppresses IL-6-responsive multiple myeloma cells. Immunity 2004:20:205-18.

18. Ji HG, Piao JY, Kim SJ, Kim DH, Lee HN, Na HK, et al. Docosahexaenoic acid inhibits Helicobacter pylori-induced STAT3 phosphorylation through activation of PPAR $\gamma$. Mol Nutr Food Res 2016;60:1448-57. 